\title{
Suppression of Thermally Excited Capillary Waves by Shear Flow
}

\author{
Didi Derks, ${ }^{1}$ Dirk G. A. L. Aarts, ${ }^{2,3}$ Daniel Bonn, ${ }^{3,4}$ Henk N. W. Lekkerkerker, ${ }^{2}$ and Arnout Imhof ${ }^{1}$ \\ ${ }^{1}$ Soft Condensed Matter, Debye Institute, Utrecht University, Princetonplein 5, 3584 CC Utrecht, The Netherlands \\ ${ }^{2}$ Van 't Hoff Laboratory, Debye Institute, Utrecht University, Padualaan 8, 3584 CH Utrecht, The Netherlands \\ ${ }^{3}$ Laboratoire de Physique Statistique, Ecole Normale Supérieure, 24 Rue Lhomond, F-75231 Paris Cedex 05, France \\ ${ }^{4}$ van der Waals-Zeeman Institute, University of Amsterdam, Valckenierstraat 65, 1018 XE Amsterdam, The Netherlands
}

(Received 31 March 2006; published 18 July 2006)

\begin{abstract}
We investigate the thermal fluctuations of the colloidal gas-liquid interface subjected to a shear flow parallel to the interface. Strikingly, we find that the shear strongly suppresses capillary waves, making the interface smoother. This phenomenon can be described by introducing an effective interfacial tension $\sigma_{\text {eff }}$ that increases with the shear rate. The increase of $\sigma_{\text {eff }}$ is a direct consequence of the loss of interfacial entropy caused by the flow, which affects especially the slow fluctuations. This demonstrates that the interfacial tension of fluids results from an intrinsic as well as a fluctuation contribution.
\end{abstract}

DOI: 10.1103/PhysRevLett.97.038301

Wind blowing across a lake causes the water surface to ripple. This rippling is resisted by both interfacial tension and gravity. The same forces act at a microscopic scale on capillary waves that exist as a result of thermal agitation [1-3]. In this Letter we provide the first visual evidence that, contrary to what happens for wind driven waves, flow strongly suppresses thermal interfacial fluctuations. To explain this, we present a simple model based on the idea that shear mostly affects the slow modes, since these couple the strongest to the flow. The observed interface smoothening will have repercussions for the understanding of the flow in, for example, micro- and nanofluidics [4] and during the process of droplet coalescence [5]. In addition, our findings are relevant to studies of shear-induced phase transitions in lamellar systems [6-10].

Thermal capillary waves have been studied extensively in molecular fluids using light [11] and x-ray scattering [12]. Recently they have also been visualized directly with a confocal microscope in a phase separated colloidpolymer mixture [13], where excellent agreement with capillary-wave theory was found. This model system has the unique property that the interfacial tension is extremely low: on the order of $10^{-9}-10^{-6} \mathrm{~N} / \mathrm{m}$ [13-16]. Thus, the amplitudes of the waves are much larger (micrometers versus nanometers) than those on the interface of molecular liquids, while their in-plane correlation length is much shorter (micrometers versus millimeters). Here, we use this system to investigate the effect of a shear flow on a freely fluctuating interface with a recently developed counterrotating shear cell [17] in real space.

We used fluorescently labeled poly(methyl methacrylate) (PMMA) colloidal spheres with radius $71 \mathrm{~nm}$ and size polydispersity less than $10 \%$ dispersed in decalin. The polymer was polystyrene (Fluka, molecular weight $2 \times$ $10^{6} \mathrm{~g} / \mathrm{mol}$ ) with radius of gyration $43 \mathrm{~nm}$. Samples were prepared by mixing stock dispersions. The phase diagram of this mixture has been determined before [13]. In the present work we used two compositions in the two-phase region of the phase diagram; one was close to the critical
PACS numbers: 82.70.Dd, 68.03.Cd, 68.03.Kn

point (sample A), the other farther removed (sample B). A sample was loaded into the shear cell, which was placed on a Leica TCS-SP2 inverted confocal scanning microscope equipped with a $100 \times 1.4$ NA oil immersion objective, and allowed to fully phase separate for $24 \mathrm{~h}$. For details of the setup and its performance, we refer to Ref. [17]. Briefly, the shear cell is a counterrotating cone-plate cell. The bottom plate consisted of a $6 \mathrm{~cm}$ diameter No. 1 cover slip, while the metal cone had an angle of $1^{\circ}$. By rotating them in opposite directions, a simple shear flow was created with a (nearly) horizontal plane of zero velocity (ZVP). Objects in this plane remain stationary with respect to the lab frame while shearing. The vertical position of the ZVP was carefully adjusted to the horizontal gas-liquid interface (at a height of $\sim 50 \mu \mathrm{m}$ above the glass plate) by varying the relative rotational velocities of cone and plate. Thus, a fixed section of the interface remains in focus during the experiment. This is essential for visualizing details of the interface without any blurring caused by the motion. At each shear rate a time series was recorded of the flow-gradient plane for typically $20 \mathrm{~min}$ at a frame rate of $1.5 \mathrm{~Hz}(0.67 \mathrm{~s} /$ frame). The vertical location of the interface $h(x)$ is determined for each column of pixels in a frame by fitting the pixel value $I(z)$, which is proportional to the local colloid concentration, to a van der Waals profile $I(z)=a+b \tanh \{[z-h(x)] / c\}$. The position thus found is shown in Fig. 1 and is seen to describe the interface well.

Snapshots of the interface in a phase separated colloidpolymer mixture are shown in Fig. 1. The lower ("liquid") phase is dense in fluorescent colloids and thus appears bright, while the upper ("gas") phase is poor in colloids. The particles are too small to be resolved individually by the microscope, but the rough interface separating the phases can be clearly observed. Although the applied shear is too small to significantly affect the thermal motion of individual particles $\left(\mathrm{Pe}=\eta \dot{\gamma} d^{3} / k_{B} T<0.1\right.$ for all experiments), the interfacial roughness is visibly reduced as the shear rate $\dot{\gamma}$ is increased. This situation is stationary in time. Turning off the shear immediately restores the equi- 


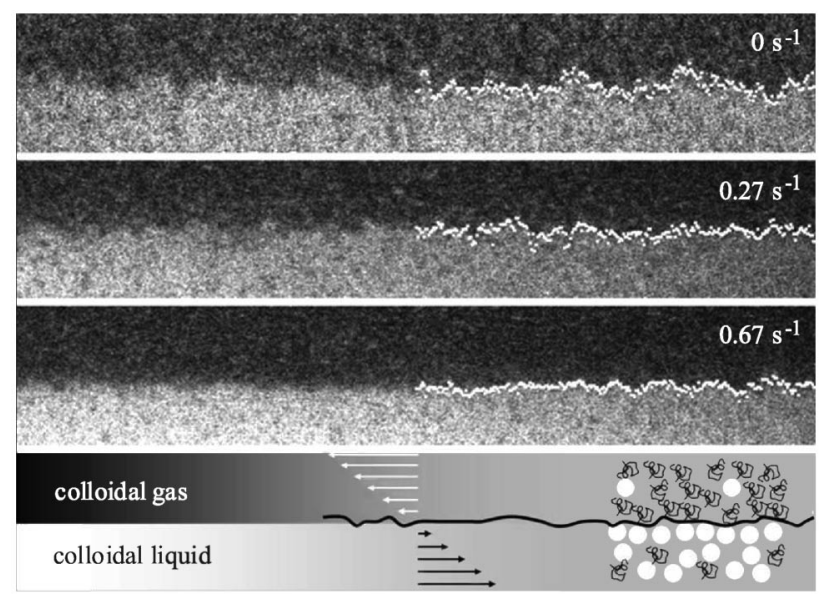

FIG. 1. Snapshots of the interface of sample A, closest to the critical point, at three shear rates. Each image is $18 \times 106 \mu \mathrm{m}^{2}$ and shows a vertical cross section through the interface. The position of the interface is indicated with bright pixels. The bottom panel schematically shows the flow geometry with the plane of zero velocity located at the interface.

librium fluctuations. We determine the vertical location of the interface $h(x)$ at each position along the horizontal $(x)$ axis as described above and obtain the probability distributions of the interface height for different shear rates [Figs. 2(a) and 2(b)]. Under shear, these remain Gaussian but have a reduced width reflecting the strong suppression of the roughness. This is confirmed in Figs. 2(c) and 2(d) where the mean-square interfacial height $\left\langle h^{2}\right\rangle$ is plotted against the shear rate. Sample B, farther from the critical point, shows a smaller roughness than $\mathrm{A}$ and requires larger shear rates to reduce the roughness.
Next, we calculate the autocorrelation function of the interface height $g_{h}(x)=\left\langle h\left(x+x^{\prime}\right) h\left(x^{\prime}\right)\right\rangle$, where the angular brackets denote averaging over the primed quantities. For a nonsheared system the theory of independent capillary-wave fluctuations [18] predicts

$$
g_{h}(x)=\frac{k_{B} T}{2 \pi \sigma} K_{0}(x / \xi)
$$

where $K_{0}$ is the modified Bessel function of the second kind. The interfacial tension $\sigma$ determines the mean-square height, or roughness, of the interface, and the in-plane correlation length $\xi$ equals the capillary length $L_{\text {cap }}=$ $\sqrt{\sigma / g \Delta \rho}$, with $g$ the acceleration of gravity and $\Delta \rho$ the density difference between the phases. From a fit we find these equilibrium properties for the two state points under study (Table I).

Interestingly, we find that for sheared interfaces the shape of the correlation function also obeys Eq. (1), only with different parameters characterizing its decay (Fig. 3). This leads to the surprising conclusion that, in the experiment, the interface under shear cannot be distinguished from an equilibrium interface with a different interfacial tension. Hence, in the sheared systems, we can assign an effective interfacial tension $\sigma_{\text {eff }}$ and a correlation length $\xi$, which is not necessarily equal to the capillary length. Clearly, both the effective interfacial tension and the correlation length increase significantly with shear rate (Fig. 4).

Why shear increases the measured interfacial tension can be understood as follows. The interface will start to feel the presence of a shear when the applied shear rate approaches the relaxation rate of the fluctuating waves. We determine the lifetime of the capillary-wave fluctuations
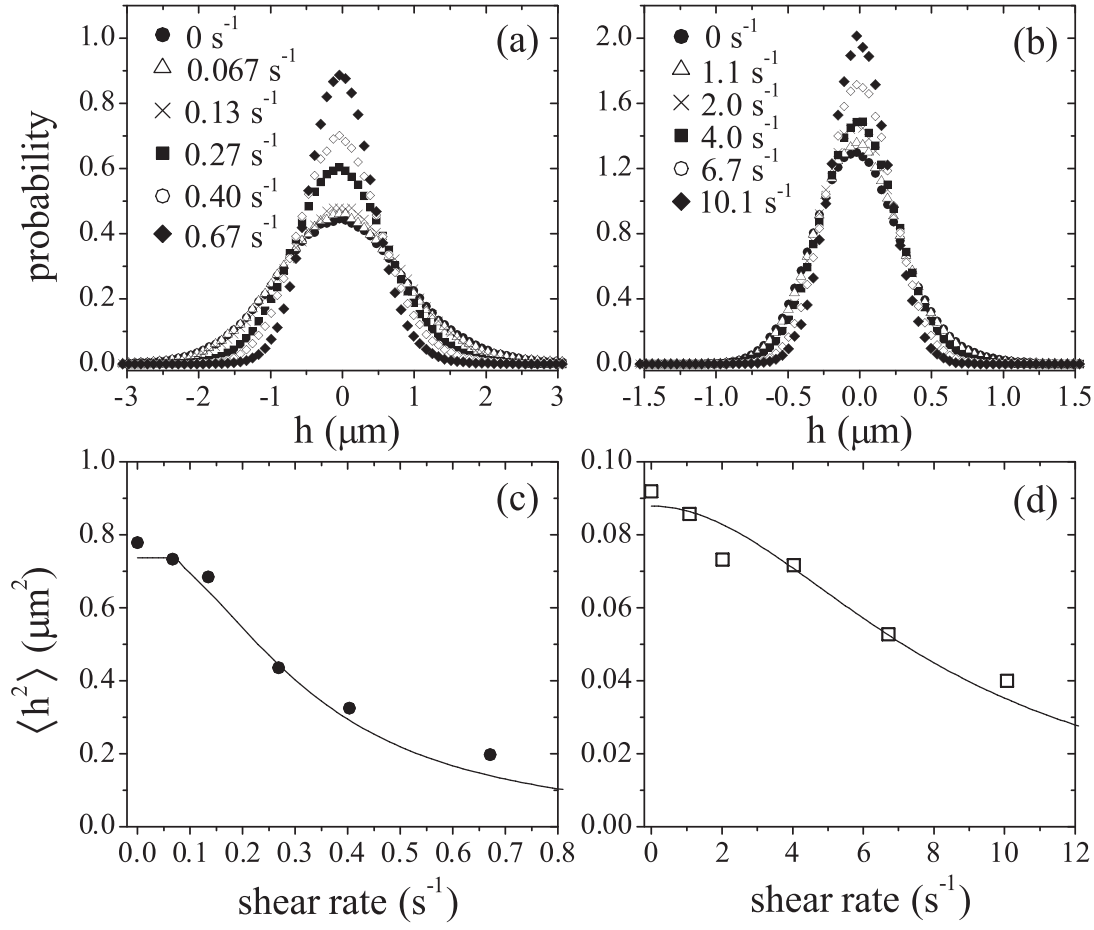

038301-2
FIG. 2. The interfacial roughness at different shear rates. The panels on top display the height distribution for (a) state point $\mathrm{A}$ and (b) state point $\mathrm{B}$ farther from the critical point. In (c) and (d) the corresponding mean-square interfacial height is plotted as function of the shear rate. The solid curves follow from the model discussed in the text. 
TABLE I. Physical properties of the equilibrium system at state point $\mathrm{A}$, near the critical point, and state point $\mathrm{B}$, away from the critical point. The interfacial tension $\sigma$, capillary length $L_{\text {cap }}$, and capillary time $\tau_{\text {cap }}$ are directly obtained from the correlation functions of the interface height at zero shear. The density difference $\Delta \rho$ and viscosity $\eta$ are calculated from these values.

\begin{tabular}{lccc}
\hline \hline & & State point A & State point B \\
\hline$\sigma$ & $(\mathrm{nN} / \mathrm{m})$ & 2.5 & 36 \\
$L_{\text {cap }}$ & $(\mu \mathrm{m})$ & 2.6 & 8 \\
$\tau_{\text {cap }}$ & $(\mathrm{s})$ & 13 & 6 \\
$\Delta \rho$ & $(\mathrm{g} / \mathrm{mL})$ & 0.038 & 0.057 \\
$\eta$ & $(\mathrm{mPa} \mathrm{s})$ & 13 & 28 \\
\hline \hline
\end{tabular}

from the dynamical height autocorrelation functions $g_{h}(t)=\left\langle h\left(t+t^{\prime}\right) h\left(t^{\prime}\right)\right\rangle$ at zero shear, as described previously [13]. Capillary-wave theory in the overdamped regime predicts [19] that a mode with wave vector $q$ decays exponentially in a time

$$
\tau_{q}=\tau_{\text {cap }} \frac{2 q L_{\text {cap }}}{1+q^{2} L_{\text {cap }}^{2}} .
$$

Here the capillary time $\tau_{\text {cap }}$ equals $\eta L_{\text {cap }} / \sigma$ and $\eta$ is the sum of the viscosities of the two phases. The experimentally obtained capillary times are listed in Table I. We thus expect that if $\dot{\gamma} \tau_{\text {cap }}>1$, the fluctuations begin to be affected. Solving $\dot{\gamma} \tau_{q}>1$ leads to two solutions, $q_{1}$ and $q_{2}$, bounding the range of wave numbers affected by shear.
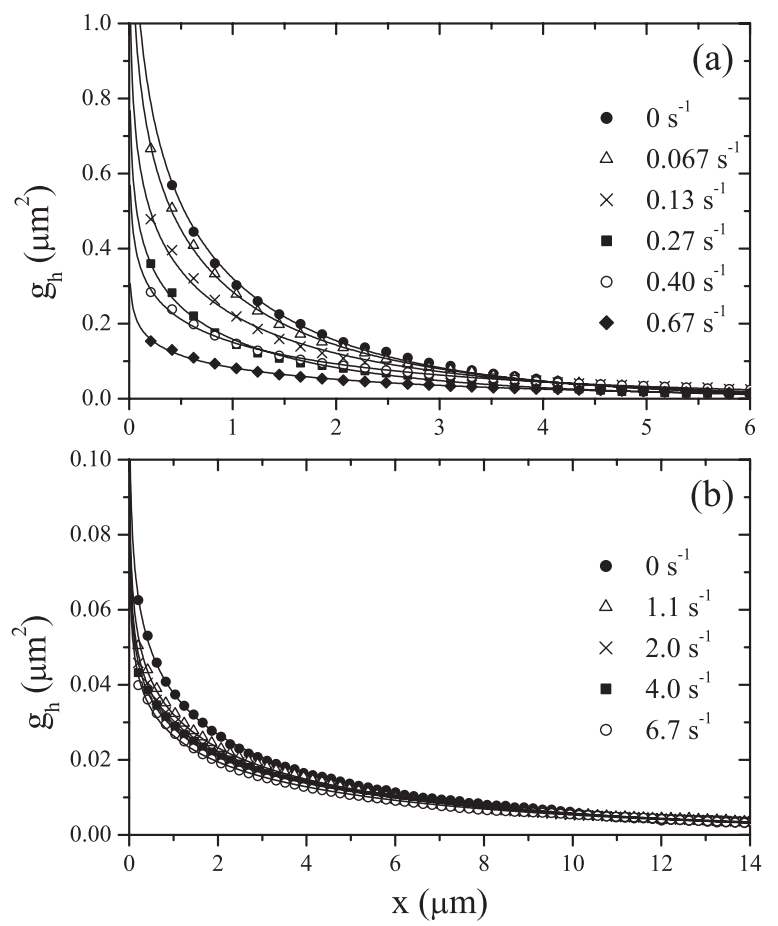

FIG. 3. The experimentally obtained static height correlation function at different shear rates for (a) state point A and (b) state point B. Symbols refer to the same shear rates as in Fig. 2. The lines are fits to Eq. (1).
How do these shear-affected waves translate into an effectively higher interfacial tension? The usual model of a fluid interface is that of an intrinsic profile, as calculated, for instance, in the mean-field theory of van der Waals [20], supplemented with fluctuations described by capillarywave theory [2,3]. Buff, Lovett, and Stillinger [21] find that the measurable interfacial tension $\sigma$ is then a sum of a "bare" interfacial tension $\sigma_{0}$ and a negative contribution to the surface free energy due to the entropy of the capillary-wave fluctuations:

$$
\sigma=\sigma_{0}-\frac{3}{16 \pi} k_{B} T q_{\max }^{2}
$$

Here, $q_{\max }$ corresponds to a microscopic cutoff length. This result can be found by assigning $\frac{1}{2} k_{B} T$ to each mode using the equipartition theorem and subsequently integrating over all modes. In case of shear, we assume that the shear-affected waves no longer contribute to the entropic lowering of the interfacial tension. If we then perform the integration leading to Eq. (3) and exclude the range $q_{1}<$ $q<q_{2}$, this results in a smaller reduction of the interfacial tension. The effective interfacial tension that follows from this calculation is

$$
\sigma_{\text {eff }}(\dot{\gamma})=\sigma+\frac{3 k_{B} T}{4 \pi} \frac{\dot{\gamma} \tau_{\text {cap }}}{L_{\text {cap }}^{2}} \sqrt{\left(\dot{\gamma} \tau_{\text {cap }}\right)^{2}-1},
$$

with $\sigma$ the interfacial tension at zero shear. All parameters
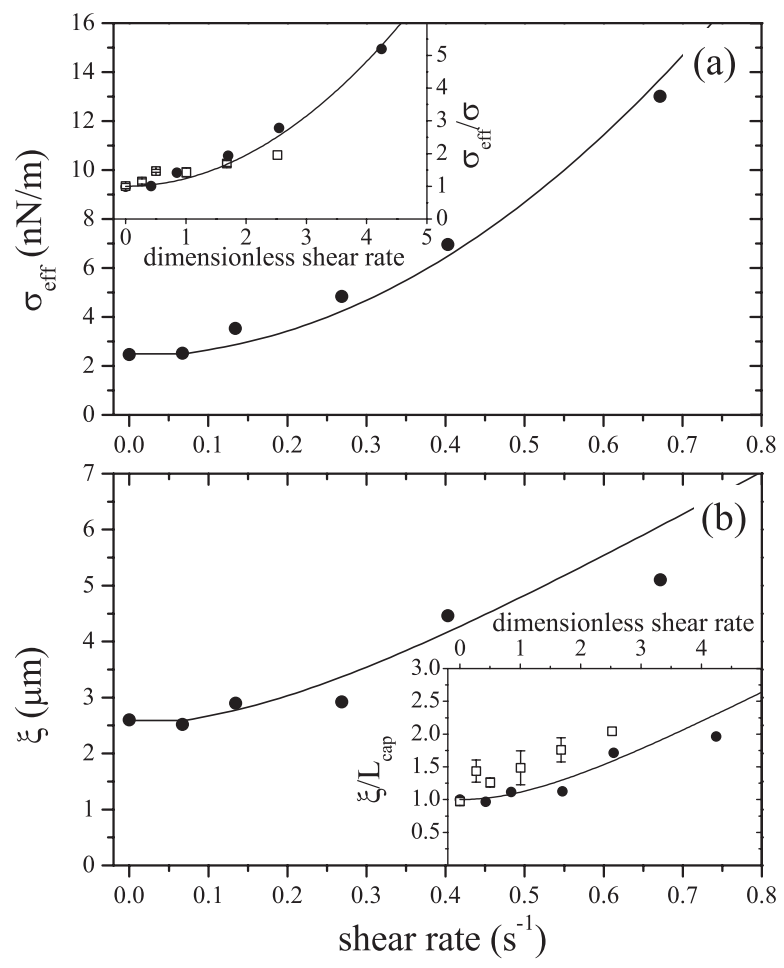

FIG. 4. The effective interfacial tension and the correlation length along the interface. Solid circles refer to state point A and open squares to state point B. The solid curves follow from the model discussed in the text. The dimensionless shear rate is defined as $\dot{\gamma} \tau_{\text {cap }} \sqrt{\left(k_{B} T / \sigma\right)} / L_{\text {cap }}$. 
going into Eq. (4) are determined from the experiment at zero shear rate (Table I). This allows for a direct comparison with the data. Without any adjustable parameters we find very good agreement for both the state point close to the critical point and, as shown in the inset, the state point farther away from the critical point [Fig. 4(a)]. Note that the second term in Eq. (4) is independent of our choice for $q_{\max }$ and depends, approximately, quadratically on the shear rate. Interestingly, a similar quadratic increase was found in recent simulations of homogeneous nucleation of colloidal crystals under shear [22]. In our model a change in the interfacial tension will also result in an increased correlation length, since $\xi(\dot{\gamma})=\sqrt{\sigma_{\text {eff }}(\dot{\gamma}) / g} \bar{\Delta} \rho$, where the density difference between the two phases is assumed to be the same as in equilibrium. Again, this is seen to be the case for state point A [Fig. 4(b)]; for state point B (inset) the trend is similar while the departure is possibly due to difficulties of determining a relatively large $L_{\text {cap }}$ in a small observation window. Since the mean-square interfacial roughness is inversely proportional to the interfacial tension $\left(\left\langle h^{2}\right\rangle_{\dot{\gamma}=0} \propto k_{B} T / \sigma\right)$, the model predicts that the amplitude of the capillary waves depends on the shear rate as $\left\langle h^{2}\right\rangle(\dot{\gamma})=\left[\sigma / \sigma_{\text {eff }}(\dot{\gamma})\right]\left\langle h^{2}\right\rangle_{\dot{\gamma}=0}$ [Figs. 2(c) and 2(d)]. Here we ignore a small logarithmic correction caused by the change in correlation length. We find that this simple calculation provides excellent quantitative agreement with the experimental data, without any adjustable parameters. Remarkable is the huge impact of shear when approaching the critical point. According to our model, a lower interfacial tension, a longer capillary time, and a shorter capillary length all work to increase $\sigma_{\text {eff }} / \sigma$. Experimentally, this is exactly what we find, leading to a reduction of the mean-square roughness of up to a factor 5 .

In conclusion, we find that a shear flow reduces thermal capillary waves, in clear contrast with wind driven waves. A similar phenomenon is predicted to play a key role in flow-induced phase transitions in membrane systems [610]. We show that the suppression of thermal fluctuations at a fluid interface can be understood from a coupling of the flow with the fluctuations. Interestingly, the experimental correlation functions can be accurately described by the theoretical result for the equilibrium case, indicating that the fluctuation modes appear in the same relative proportions. Assuming that too slow modes, while still present, no longer contribute to the entropy of the interface allows prediction of an effective interfacial tension, correlation length, and interfacial roughness. This produces quantitative agreement with our experimental data. The success of equilibrium theory and the formulation of a more complete hydrodynamic theory of the flow-fluctuation coupling are now clearly put as theoretical challenges. These results do not depend on the specific dynamics of the particles and should apply equally to Brownian and molecular systems. Perhaps the most important conclusion is that assuming a partial suppression of the fluctuations leads to an increase in the effective surface tension. This is to our knowledge the first direct experimental confirmation of the dual nature of fluid interfaces, with a high surface tension due to the intrinsic density profile that is lowered by the entropy of the fluctuations. Further investigation is necessary to see whether our findings allow one to completely reconcile the two models, a central point for our understanding of fluid interfaces.

The authors would like to thank D. Bartolo, A. Fortini, J. Zwanikken, R. van Roij, and J. Meunier for fruitful discussions. This work is part of the research program of the "Stichting voor Fundamenteel Onderzoek der Materie (FOM)," which is financially supported by the "Nederlandse Organisatie voor Wetenschappelijk Onderzoek (NWO)." D. G. A. L. A. acknowledges funding through a Marie Curie Intra-European Fellowship. LPS de l'ENS is UMR8550 of the CNRS, associated with the universities Paris 6 and Paris 7.

[1] V. G. Levich, Physicochemical Hydrodynamics (Dekker, New York, 1962).

[2] M. von Smoluchowski, Ann. Phys. (Leipzig) 25, 205 (1908).

[3] L. Mandelstam, Ann. Phys. (Leipzig) 41, 609 (1913).

[4] M. Moseler and U. Landman, Science 289, 1165 (2000).

[5] D. G. A. L. Aarts, H. N.W. Lekkerkerker, H. Guo, G. H. Wegdam, and D. Bonn, Phys. Rev. Lett. 95, 164503 (2005).

[6] M.E. Cates and S. T. Milner, Phys. Rev. Lett. 62, 1856 (1989).

[7] R. Bruinsma and Y. Rabin, Phys. Rev. A 45, 994 (1992).

[8] J. Yamamoto and H. Tanaka, Phys. Rev. Lett. 74, 932 (1995); 77, 4390 (1996).

[9] K. A. Koppi, M. Tirrell, and F. S. Bates, Phys. Rev. Lett. 70, 1449 (1993).

[10] A. G.Zilman and R. Granek, Eur. Phys. J. B 11, 593 (1999).

[11] D. Langevin, Light Scattering by Liquid Surfaces and Complementary Techniques (Prentice-Hall, Englewood Cliffs, NJ, 1992).

[12] T. Seydel, A. Madsen, M. Tolan, G. Grübel, and W. Press, Phys. Rev. B 63, 073409 (2001).

[13] D. G. A. L. Aarts, M. Schmidt, and H. N. W. Lekkerkerker, Science 304, 847 (2004).

[14] G. A. Vliegenthart and H. N.W. Lekkerkerker, Prog. Colloid Polym. Sci. 105, 27 (1997).

[15] E. H. A. de Hoog and H. N.W. Lekkerkerker, J. Phys. Chem. B 103, 5274 (1999).

[16] D.G.A.L. Aarts, J.H. van der Wiel, and H.N.W. Lekkerkerker, J. Phys. Condens. Matter 15, S245 (2003).

[17] D. Derks, H. Wisman, A. van Blaaderen, and A. Imhof, J. Phys. Condens. Matter 16, S3917 (2004).

[18] B. R. McClain, D. D. Lee, B. L. Carvalho, S. G. J. Mochrie, S. H. Chen, and J.D. Litster, Phys. Rev. Lett. 72, 246 (1994).

[19] U.-S. Jeng, L. Esibov, L. Crow, and A. Steyerl, J. Phys. Condens. Matter 10, 4955 (1998).

[20] J. D. van der Waals, Z. Phys. Chem. 13, 657 (1894).

[21] F. P. Buff, R. A. Lovett, and F. H. Stillinger, Phys. Rev. Lett. 15, 621 (1965).

[22] R. Blaak, S. Auer, D. Frenkel, and H. Löwen, Phys. Rev. Lett. 93, 068303 (2004). 